\title{
A Multi-resolution CLS Detection Algorithm for Mammographic Image Analysis
}

\author{
Lionel C.C. Wai, Matthew Mellor, and Michael Brady \\ Medical Vision Laboratory, Robotics Research Group, University of Oxford. \\ Ewert House, Summertown, Oxford OX2 7DD, United Kingdom. \\ \{lwai, matt, jmb\} @robots.ox.ac.uk
}

\begin{abstract}
Curvilinear structures (CLS) are locally one-dimensional, relatively thin objects which complicate analysis of a mammogram. They comprise a number of anatomical features, most especially connective tissue, blood vessels, and milk ducts. The segmentation, identification and removal of such structures potentially facilitate a wide range of mammographic image processing applications, such as mass detection and temporal registration. In this paper, we present a novel CLS detection algorithm which is based on the monogenic signal afforced by a CLS physical model. The strength of the proposed modelbased CLS detector is that it is able to identify even low contrast CLS. In addition, a noise suppression approach, based on local energy thresholding, is proposed to further improve the quality of segmentation. A local energy (LE)based junction detection method which utilises the orientation information provided by the monogenic signal is also presented. Experiments demonstrate that the proposed CLS detection framework is capable of producing welllocalized, highly noise-tolerated responses as well as robust performances as compared to classical orientation-sampling approach.
\end{abstract}

\section{Introduction}

Mammographic image processing aims primarily to detect, classify, and measure anatomical features such as masses and microcalcifications, and to monitor the development of such features over time, view, and bilaterally. Amongst the anatomical features that can be seen in mammograms, curvilinear structures (CLS) are often the most pervasive but the most complex feature to segment. They correspond to relatively dense connective stroma, milk ducts, and blood vessels, and appear locally linear and thin. The detection of CLS is often difficult because of their low contrast, variable widths, and the often noisy parenchymal background against which the feature has to be detected. The projective nature of mammography further complicates detection. The detection and segmentation of CLS has been studied in various applications such as the validation of calcification detectors and registration $[1,2]$. Identifying the CLS is often a key step in distinguishing tumour spicules from overlying CLS. Some previous researchers have tackled the problem of detecting and removing the CLS. For example, Cerneaz [3] proposed a CLS detector based on spatial second dimensional derivative operators. Zwiggelaar et. al. [4] suggested an 
nonlinear line strength operator to detect the linear structures in mammograms. In this study, we extended the work of Schenk [5] on local energy (LE) feature detection which is based on a steerable filter framework.

From the perspective of low-level feature detection, the CLS segmentation problem can be regarded as a ridge detection task. However, most previous work on feature detection has been focused on the edge detection, of which has the sudden intensity change or higher local energy than the corresponding ridge. Kovesi's [6] Phase Congruency algorithm (based on previous work by Morrone and Owens) defines a feature as an image location for which the local phase remains relatively constant across a sufficient range of bandpass filters. Kovesi's approach is intrinsically one-dimensional, requiring a framework such as steerable filters to extend it to images. More recently, coupled with the Reisz transform, Felsberg [7] has developed the construction of a two or more dimensional feature detection scheme. This has significant advantages over steerable filtering both in terms of the robustness and speed of execution and isotropicity of filter responses. This paper adapts the monogenic signal to the task of CLS detection and removal.

In the remaining of this paper, the framework and the algorithms of the CLS detector are introduced. Subsequently, the effect of noise and the responses of the algorithm of CLS with different widths are showed and its performance is discussed. Finally, segmentation results on real mammographic images are presented.

\section{Segmentation Methodologies}

In this section, we introduce the CLS model, which approximates the intensity profile of a CLS feature in a digitized image, and the multi-resolution ridge detection algorithm based on the monogenic signal.

\subsection{The Digitized Intensity Profile Model of CLS}

The CLS model employed in our detection framework is adapted from the work of Cerneaz, which introduced a two medium model that estimates the intensity profile of the CLS. In this model, the CLS are assumed to have circular cross section when the breast is not compressed. Since the CLS cannot be assumed orthogonal to the beam, the cross section for mammography will be elliptical. To simplify the analysis, though not the detection process, the $\mathrm{x}$-ray beam is assumed to be monogenic, with no scatter.

Imagine that an x-ray beam goes through a compressed breast of thickness $H$ and a CLS with an elliptic cross-section area which is of radii $a$ and $b$, in which radii $a$ is parallel to the film surface. Thus the x-ray path that transverses through the cross section of the CLS can be represented by the function $h(x)$ :

$$
h(x)=\frac{2 b}{a} \sqrt{a^{2}-x^{2}}, \quad-a \leq x \leq a .
$$

Thus the net beam attenuation of the two medium model will be 


$$
E(x)=\left\{\begin{array}{cc}
E_{o} e^{-\mu_{1}(H-h(x))} e^{-\mu_{2} h(x)} & -a \leq x \leq a \\
E_{o} e^{-\mu_{1} H} & \text { otherwise }
\end{array},\right.
$$

where $E_{o}$ is the energy of the monoenergetic incident beam, $\mu_{1}$ and $\mu_{2}$ are the x-ray attenuation coefficients of the parenchyma and the CLS respectively. The basic model is depicted in Fig. 1. By a linear approximation of the film characteristic curve, we arrive at a film density function $D$ :

$$
D=\gamma \log _{10}(\beta E),
$$

where $\beta$ is the film speed and $\gamma$ is the film gradient.

Based on this relationship, the pixel intensity profile on a digitised image can be modeled, depending on which of two main categories of digitisation method is used: film density and transmitted-light. For film density direct digitisation, the pixel intensity profile is well approximated by $P_{d}$ :

$$
P_{d}(x)=\frac{m \gamma}{\ln 10}\left[\ln \left(\beta \phi X_{c} \varepsilon_{p}\right)-\mu_{1} H-\left(\mu_{2}-\mu_{1}\right) \frac{2 b}{a} \sqrt{a^{2}-x^{2}}\right]+q,-a \leq x \leq a .
$$

Correspondingly, for transmitted-light, the profile is calculated by the equation $P_{l}$ which is showed below:

$$
P_{l}(x)=\frac{a I_{l}}{\left(\beta \phi X_{c} \varepsilon_{p}\right)^{\gamma}} \exp \left[\gamma \mu_{1} H+\gamma\left(\mu_{2}-\mu_{1}\right) \frac{2 b}{a} \sqrt{a^{2}-x^{2}}\right]+\lambda,-a \leq x \leq a .
$$

In the two equations above, $\gamma$ represents the film gradient, $\varepsilon_{p}$ is the photon density, $\mathrm{m}$ and $\mathrm{q}$ are the gradient and offset of the linear approximation of the film density digitalisation curve respectively, and $\beta \varphi X_{c} \varepsilon_{p}$ are the imaging parameters which are functions of $\mathrm{H}$ and $\mu_{1}$. And in this study, $0.6 \quad D \quad 3.0,0 \quad P \quad 255, \gamma=3, \varepsilon_{p}=17.4$, $\mu_{1}=0.558, \mu_{2}=1.028, H=3.5$.
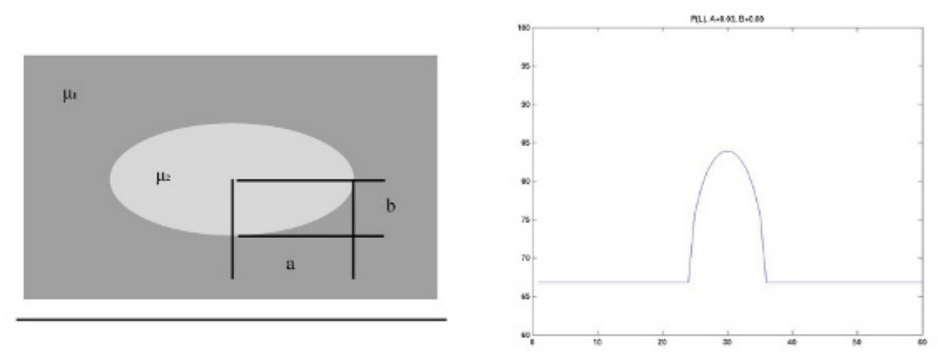

Fig. 1. The cross section of a CLS and its intensity profile. Left: The CLS model parameters as described in section 2.1. Right: An example of the intensity profile generated by the model, which is through transmitted-light digitalisation. The maximum pixel intensity is set to be 256 . 


\subsection{The Multi-resolution Ridge Detector}

A multi-resolution approach is opted for because of the variable widths of vascular structures. The CLS in mammogram are composed of large connective stroma, arteries and veins as well as narrow capillaries and milk ducts, which covers a set of vessels with width ranges from 1800 microns to 5 microns. However, in view of current limits on sampling and signal-to-noise, we are only interested in detecting the structures of widths between 1800 microns to 180 microns. This specification is enough to cover most vascular structures in mammograms sampled at as fine as 50 microns per pixel.

From the model described in section 2.1 and visualized in Fig. 1, the response of the filter should be localised at the ridges of CLS in order to get the "skeleton" extracted. In this study, we adopt the notion of phase congruency (PC) in detecting features [6]. This is a measure of the phase similarity of the Fourier components of a point. Phase congruency of a point with spatial coordinate given by $x$ can be defined as follows:

$$
P C(x)=\operatorname{Max}_{\theta \in(0,2 \pi)} \frac{\int_{-\infty}^{\infty} a_{\omega} \cos \left(\omega x+\phi_{\omega}-\theta\right) d \omega}{\int_{-\infty}^{\infty} a_{\omega} d \omega} .
$$

In order to compute PC, the local phase of the pixel must be estimated from the analytical signal. In previous work, including that by Schenk [5], a set of steerable filters are designed to estimate the local phase by analysing one dimensional cross sections of the image at each point in several different orientations. This intrinsically computationally intensive approach stems from the fact that the analytic signal, the basis for local phase of a signal, is only defined for one dimension signals. However, by using vector filters as suggested by Felsberg [7], a quadrature filter triple for the image can be obtained. The odd filter is based on the Reisz transform $H\left(u_{1}, u_{2}\right)=\left(H_{1}, H_{2}\right)$, in which

$$
H_{1}\left(u_{1}, u_{2}\right)=i \frac{u_{1}}{\sqrt{u_{1}^{2}+u_{2}^{2}}} \text { and } H_{2}\left(u_{1}, u_{2}\right)=i \frac{u_{2}}{\sqrt{u_{1}^{2}+u_{2}^{2}}} .
$$

In this expression $u_{1}$ and $u_{2}$ are the Fourier variables. This leads to a generalization of the analytical signal, which is called the monogenic signal and which can be described by the following formula:

$$
f_{M}\left(x_{1}, x_{2}\right)=\left(f\left(x_{1}, x_{2}\right),\left(h_{1} * f\right)\left(x_{1}, x_{2}\right),\left(h_{2} * f\right)\left(x_{1}, x_{2}\right)\right),
$$

where $f$ is an appropriate filter or filter bank. For multi-resolution image processing, those filters constitute a family which tries to analyse the image from different perspective. One of the most common filter sets is the difference of two Gaussian kernels, which has the advantage of effective analysis in both one- and twodimensional domains.

Based on the idea of ridge detection which is built on the support of monogenic signal and phase congruency, the degree of scale span of the multiresolution filters are designed based on the tuning on a set of CLS with certain widths. For example, if the 
CLS identified are then removed, the CLS width range should be as wide as possible. On the other hand, if CLS segmentation is for registration purposes, only salient and large vessels are needed so that the CLS detector should be biased towards CLS in the higher scale. Thus the CLS model can improve the specificity and selectivity of the detector under different applications.
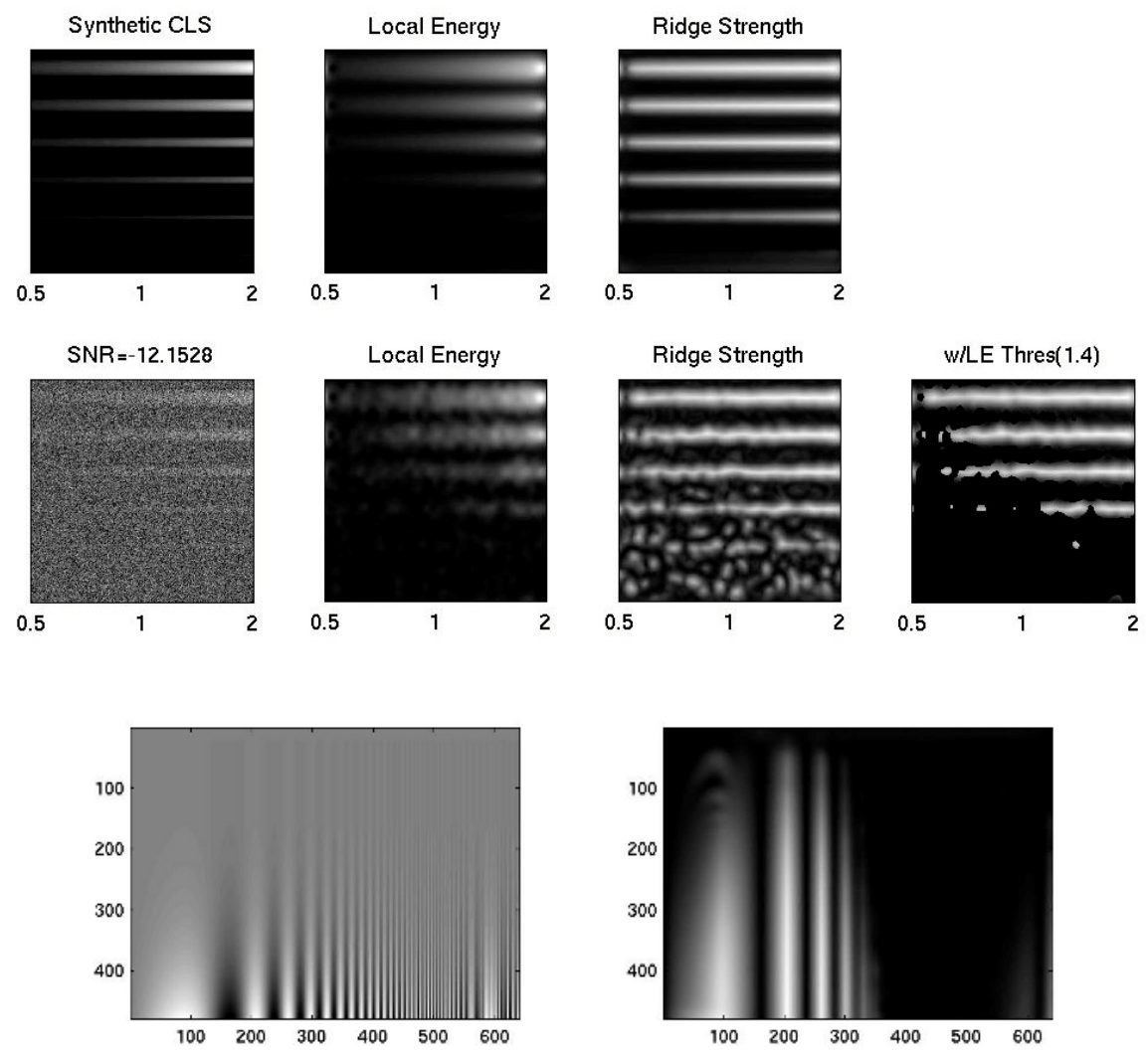

Fig. 2. Performance of the CLS detector evaluation. Upper graph: The ridge strength (phase congruency and the phase angle compound measure) of the 50 micron detector against 6 synthetic CLS with widths 180, 504, 828, 1152, 1476 and 1800 microns. The contrast on each individual synthetic CLS is varied by the $a-b$ ratio: the ratio of the horizontal and vertical radii of the CLS shown in both Fig. 1 and Eq. 1. The a-b ratio ranges from 0.5 to 2 as depicted. Middle graph: The performance of the detector against same synthetic CLS as upper graph with noise added, and the performance of the detector with noise suppression by local energy (LE) thresholding strategy. Lower graph: The performance of the detector compare with that of human visual system. The left image is the original CSF image, and the response of the CLS detector is shown on the right. 


\section{Noise Suppression and Junction Detection}

Another problem is noise. As CLS are typically low contrast and poor signal-to-noise can badly affect the performance of the CLS detector, as one can see in Fig. 2. In the proposed framework, we use local energy (LE) thresholding [8] to suppress the undesirable response from noise. The local energy is related to phase congruency, in discrete terms, as follows:

$$
\operatorname{LE}(x)=P C(x) \sum_{n} A_{n},
$$

where $A_{n}$ is the amplitude of the nth bandpass filter. The performance of LE thresholding is showed in Fig. 2.

It is well-known that phase congruency is sensitive to noise as it is the trade-off of its high sensitivity of features of low contrast. However, by thresholding LE, it is found that the false positives can be suppressed. Moreover, the response of the detector to CLS with widths over 1000 micron is still perfect even under a SNR as low as -12. (Fig. 2)
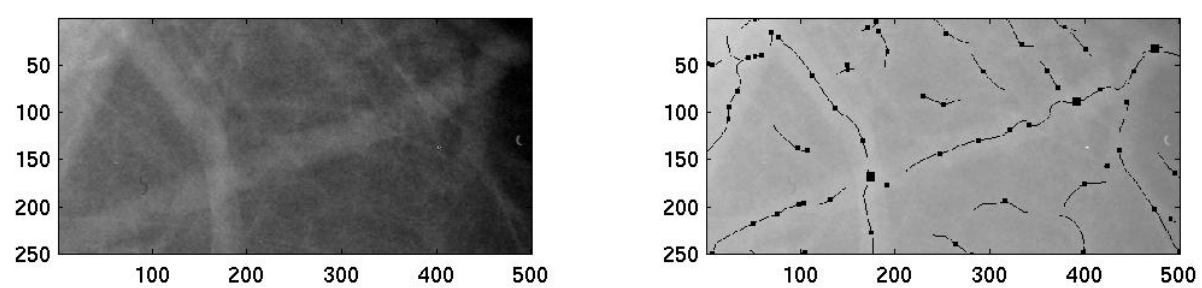

Fig. 3. Sample output of the junction detection algorithm. Larger marks are the affirmed junctions, which are inferred based on the neighbourhood orientation information and local energy. The smaller ones are rejected candidates (i.e. number of branches $<=2$ ).

Apart from noise removal, the local energy is also used to determine CLS junctions. The detection of CLS junctions is based on a two-step process. The first step is to find those pixels which have a local maximum of local energy, based on a notion that the convergence, or intersection, of ridges will result in a point with high local energy. In other words, we try to find junctions that are more "salient" than their branches. In our approach, we search the CLS skeletons to find the local maxima of LE, this will end up with a set of candidate points. In the second step, these candidate points are then searched through a neighbourhood of radius $r$, where $r=k L E(x)$. We search inside the neighbourhood to find any CLS (branches) that point towards that candidate point, by comparing the orientation of the CLS and the vector pointing to the branch from the junction point. The orientation information can be computed from the monogenic signal. A junction is detected if the number of branches is more than 2. Some typical results of junction detection are shown in Fig. 3. 


\section{Performance Evaluation}

We have applied our CLS detector to a set of mammograms and some of the results are shown in Fig. 4. In our tests, we have used mammograms digitised at resolutions of $50 \mathrm{microns} / \mathrm{pixel}$ and $300 \mathrm{microns} / \mathrm{pixel}$.

In addition, we are interested in comparing the capability of our CLS detector with the capability of human vision (Fig. 2). A Contrast Sensitivity Function (CSF) test image, which was originally a tool to study how the human visual system responds to different contrast and spatial frequency [9], is used to test the responses of our feature detector. Two findings can be derived from the test. Firstly, the dome-shaped response resembles the human visual system. Secondly, the sensitivity of the detector in low contrast regions in specific spectra is comparable to that of the human visual system and may even surpass it. This facts further support that appropriate usage of such a segmentation algorithm might enhance the ease of feature detection tasks for radiologists.

Some of the sample results carried out on real images are displayed in Fig. 4. As can be seen, the detector gives well-localized and contrast-insensitive responses on most of the weak ridges of the CLS and is not sensitive to two-dimensional structures, for example, masses. As shown in the figure, the CLS detector does not give false responses to the edges of the mass and the boundary of the pectoral muscle. Also, in regions with rapidly varying contrasts, for example, across the pectoral muscle and near the breast boundary, the response of the detector is still strong and welllocalized. Some discontinuities can be observed from the response, which can be solved by applying further processing techniques like Hough transform or by morphological operations.
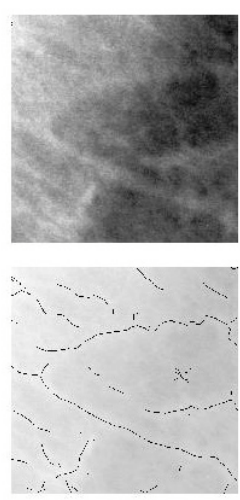
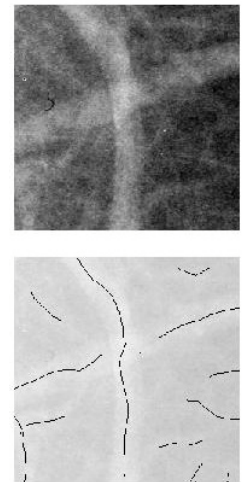
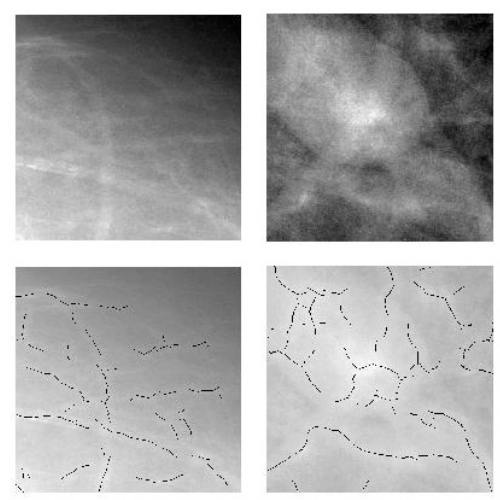

Fig. 4. Some results of the CLS detector on mammograms (digitized at a resolution of 50 micron per pixel). Here shows the response of the CLS detector in different parts of the mammogram. From left to right: 1. a CLS crossing between the parenchyma and the pectoral muscle; 2. a typical X-junction; 3. a region near the breast boundary; 4. a region around a mass. All the parameters of the CLS detector is identical for these response demonstrated. 


\section{Conclusions}

We have proposed a CLS ridge detection algorithm in this paper. Departing from previous approaches, this algorithm is based on the monogenic signal. As the segmentation is phase-based, the algorithm is able to detect CLS in low-contrast. When equipped with LE noise suppression, it has proven to be highly effective in noise removal. A CLS intensity profile model has been incorporated into the design to provide utilities for scale tuning and performance evaluation. In addition, the performance of the CLS detector is compared with the capability of human vision system by using a CSF image, which shows comparable performance of our design with the naked eye. Overall, we showed that our novel CLS detection strategy is superior in aiding or enhancing the potential, or current, CLS detection and segmentation tasks in the industry, such as employing CLS junctions as landmarks in mammogram registration, enhancing the reliability of mass detection by CLS removal, or reduction of the false positives in microcalcifications detection (in which most false positives are indeed intersections of capillaries). Moreover, as onedimensional vascular structures (e.g. blood vessels, lymph nodes) are pervasive anatomical entities itself, we hope that our algorithm can be applied to linear structure identification in other x-ray image processing and even to other modalities.

Acknowledgements. Lionel C. C. Wai is supported by the Croucher Foundation, Hong Kong; Matthew Mellor is supported by the MIAS Interdisciplinary Research Consortium funded by the EPSRC and MRC.

\section{References}

1. N. Vujovic, "Establishing the correspondence between control points in pairs of mammographic images," IEEE Transactions on Image Processing, vol 6, no. 10, ppl 13881399, 1997.

2. R. Marti, R. Zwiggelaar, C. Rubin, “Automatic registration of mammograms based on linear structures", in Information Processing in Medical Imaging (IPMI 2001), pp. 162-168, LNCS 2082, Spinger-Verlag, 2001.

3. N. Cerneaz, "Model-based analysis of mammograms", DPhil Thesis, University of Oxford, 1994.

4. R. Zwiggelaar, T. Parr, C. Taylor, "Finding orientated line patterns in digital mammographic images", in Proceedings $7^{\text {th }}$ British Machine Vision Conference, pp. 715-724, 1996.

5. V. U. B. Schenk, M. Brady, "Finding CLS using multiresolution oriented local energy feature detection," in Proceedings $6^{\text {th }}$ International Workshop on Digital Mammography (IWDM 2002), June 2002.

6. P. Kovesi, "Image features from phase congruency", Videre: A Journal of Computer Vision Research, Vol.1, No.3, 1999.

7. M. Felsberg, G. Sommer, "A new extension of linear signal processing for estimating local properties and detecting features", in Proceedings of DAGM Symposium, Spinger-Verlag, pp. 195-202, 2002.

8. V. U. B. Schenk, "Visual identification of fine surface incisions", DPhil Thesis, University of Oxford, 2001.

9. F. W. Campbell, J. B. Robson, "Application of Fourier analysis to the visibility of gratings", Journal of Physiology, vol. 197, pp. 551-566, 1968. 